\title{
Longitudinal surveillance of SARS-like coronaviruses in bats by quantitative real-time PCR
}

\section{Dear Editor,}

The 2002-2003 severe acute respiratory syndrome coronavirus (SARS-CoV) (Drosten et al., 2003) caused human pandemics that began in China and spread globally. Subsequently, diverse SARS-like coronaviruses (SL-CoVs) have been detected in horseshoe bats in China, Europe, and Africa (Li et al., 2005; Tong et al., 2009; Drexler et al., 2010). Recently, we found SL-CoVs with high genetic diversity in a single bat colony predominantly roosted by Chinese horseshoe bats (Rhinolophus sinicus) in Kunming, Yunnan province (Ge et al., 2013). Two of these SL-CoVs are able to use human ACE2 as a receptor for cell entry (Ge et al., 2013; Menachery et al., 2015), highlighting the risk of this group of viruses to humans and the importance of long-term surveillance.

We conducted a longitudinal surveillance study of bat SL-CoVs using quantitative real-time PCR (qRT-PCR) targeting the nucleocapsid $(N)$ and RNA-dependent RNA polymerase $(R d R p)$ genes in one bat population in Yunnan, China. A total of 431 bat fecal samples were collected during 2011-2014. Total RNA extraction was performed with $200 \mu \mathrm{L}$ of each fecal sample using a High Pure Viral RNA Kit (Roche, Basel, Switzerland) according to the manufacturer's instructions. Five microliters of RNA was used to screen for all alphacoronaviruses and betacoronaviruses as previously described (Ge et al., 2013). To construct standard templates, fragments of the $N$ and $R d R p$ genes were amplified from WIV1 genomic RNA and cloned into the pGEM-T Easy Plasmid Vector (Promega, Madison, USA). The primers and probe for the $N$ gene were adopted from our previous report (Ge et al., 2013) and the primers and probe targeting the conserved region of $R d R p$ was newly designed (Supplementary Table $\mathrm{S} 1$ ). Both forward primers contained a $5^{\prime}-\mathrm{T} 7$ RNA polymerase promoter sequence (TAATACGACTCACTATAGGG) to facilitate in vitro transcription. Correct clones were transcribed using the MAXIscript ${ }^{\circledR}$ Kit (Applied Biosystems, Waltham, USA). Following purification and quantification, ten-fold serial dilutions of the RNA transcripts of the $N$ and $R d R p$ genes were used as external standards to calculate viral concentrations, which are expressed as genome copies per gram of bat feces (copies/g).

The qRT-PCRs were performed using an AgPathID $^{\text {TM }}$ One-Step RT-PCR Kit (Applied Biosystems) according to the manufacturer's instructions. Each $25-\mu \mathrm{L}$ reaction mixture contained $12.5 \mu \mathrm{L}$ of $2 \times$ RT-PCR buffer, $1 \mu \mathrm{L}$ of RT-PCR Enzyme Mix, $400 \mathrm{nmol} / \mathrm{L}$ each primer, $120 \mathrm{nmol} / \mathrm{L}$ probe primer, and $1 \mu \mathrm{L}$ of nucleic acid extract. Amplification was carried out in 96-well plates using the StepOne PCR system (Applied Biosystems). Thermocycling conditions were as follows: 10 min at $45{ }^{\circ} \mathrm{C}$ for reverse transcription, $10 \mathrm{~min}$ at $95{ }^{\circ} \mathrm{C}$ for activation of the Taq DNA polymerase, and 40 cycles of $95{ }^{\circ} \mathrm{C}$ for $15 \mathrm{sec}$ and $60{ }^{\circ} \mathrm{C}$ for $45 \mathrm{sec}$. Each run included three viral positive control templates and one negative control to evaluate assay performance. A positive result was defined as a well-defined exponential fluorescence curve that crossed the cycle threshold $(C t)$ within 38 cycles. A specimen with a $C t$ value $>36$ was assayed again to exclude operation faults. Data were analyzed using the analysis of variance (ANOVA) for continuous variables. All comparisons were two-tailed and a $P$-value of less than 0.05 was considered significant.

The analytical detection range and sensitivity of the two real-time PCR assays for SL-CoVs were investigated by testing 10 -fold serial dilutions of RNA transcripts and WIV1 genomic RNA. The highest dilution of transcripts at which all three replicates were positive was defined as the limit of detection (LoD). $C t$ values were plotted against the $\log 10$ of gene copy number, and linearity was observed over the entire virus concentration range (Supplementary Figure S1). LoD values of $N$ and $R d R p$ transcripts were three and four copies, respectively. Linear amplifications for the $N$ assay ranged from $10^{1}$ to $10^{9}$ copies/reaction (efficiency values, $132 \%$ ), while for $R d R p$ were from $10^{\circ}$ to $10^{9}$ copies/reaction (efficiency values, $109 \%$ ). The LoDs of WIV1 genomic RNA were as low as $2.04 \times 10^{-2}$ plaque forming units (pfu)/reaction for both assays. Linear amplifications ranged from $2.04 \times 10^{-2}$ to $2.04 \times 10^{5} \mathrm{pfu} /$ reaction, with efficiency values of $85 \%$ and $84 \%$, respectively (Supplementary Figure S2). The specificity of the two assays was then confirmed using Orthoreovirus isolated from a 
bat (Yang et al., 2015), and viral-containing bat fecal samples that are positive for paramyxovirus, hepatitis A virus, hepatitis B virus, hepatitis E virus, or coronaviruses other than SL-CoVs (unpublished). No false positive was observed for any of these samples (data not shown).

Fifty-seven of 431 bat fecal samples were positive for SL-CoVs by RT-PCR screening. The detection rate varied significantly among sampling dates (ANOVA, $F=$ $28.42, P=0.03$ ), from $3.1 \%$ to $48.7 \%$. The highest detection rate was observed in September 2012. Standard curves of in vitro transcribed RNA for both assays were obtained, with $R^{2}>0.99$ (Supplementary Figure S3). qRT-PCR assays for $N$ and $R d R p$ were used to quantify the concentration of SL-CoVs in the positive samples. Both assays were sensitive and efficient when extracted RNAs were used as templates. Additionally, the $C t$ values (means of triplicates \pm standard deviation) for most of the bat fecal samples were within 36 . The virus con- centrations of these specimens are listed in supplementary Table S2. Of note, the concentration of individual samples varied significantly (ANOVA, $F=4.03, P<$ $0.001)$, from $10^{5}$ to $10^{11}$ copies $/ \mathrm{g}$ bat feces in the $R d R p$ assay or $10^{6}$ to $10^{11}$ copies/g in the $N$ assay (Figure $1 \mathrm{~A}$ ). The sample with the highest virus concentration was collected in September $2012\left(1.71 \times 10^{11}\right.$ copies/g for the $R d R p$ assay and $4.58 \times 10^{11}$ copies/g for the $N$ assay. Ten samples $(17 \%)$ had virus concentrations of greater than $10^{9} \mathrm{copies} / \mathrm{g}$, four of which were collected in September 2012 and four in July 2013. Additionally, the average of bat SL-CoVs for the seven sampling times was evaluated. As shown in Figure 1B, three significant peaks were observed (ANOVA, $F=1.5, P<0.05$ ). The first peak corresponding to the highest virus detection rate was observed in September 2012. The second peak was in July 2013 and the third peak was in October 2014.

Both assays sensitively and efficiently detected SLCoV RNA in environmental samples. However, the vir-

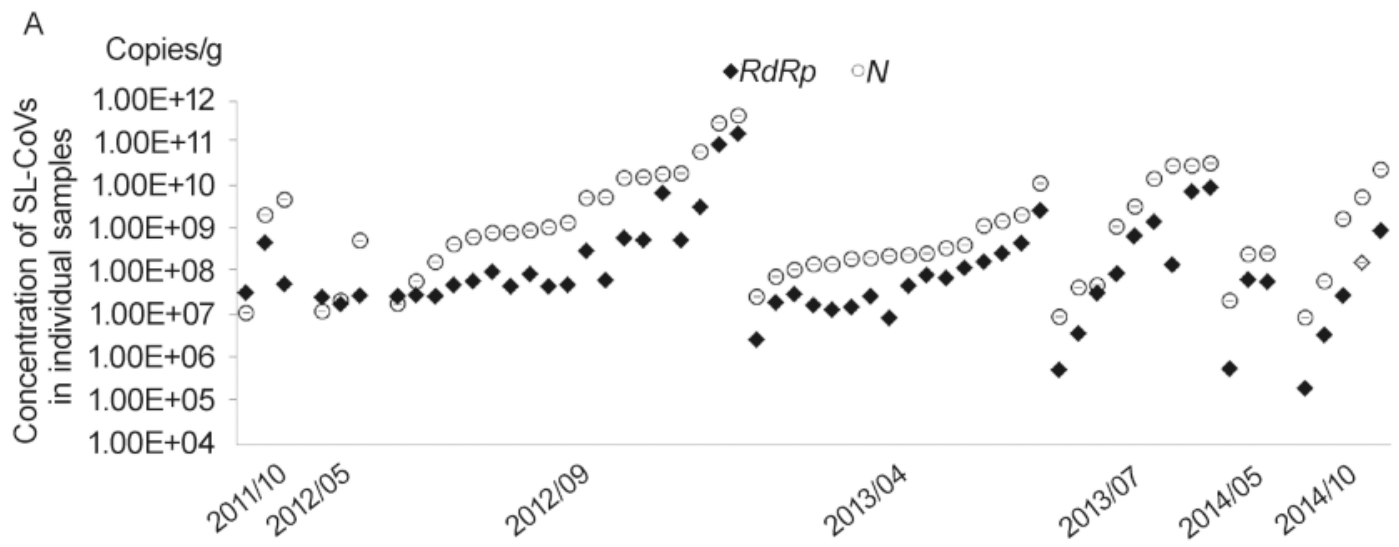

Time of samples collected

B

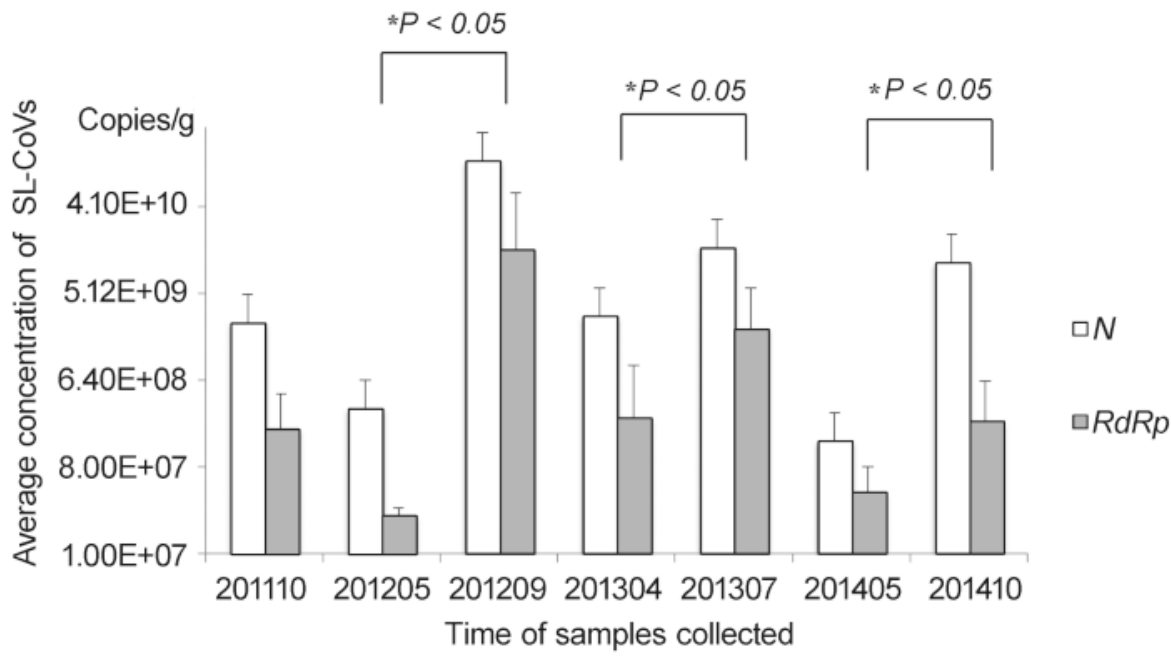

Figure 1. qRT-PCR assays of SL-CoVs in bat fecal samples. (A) SL-CoV concentration in individual bat fecal samples. Circles represent the $N$ assay, diamonds represent the $R d R p$ assay. (B) SL-CoV concentrations for seven sampling dates. 
us concentrations for the $R d R p$ assay were slightly lower than those of the $N$ assay, normally by one order of magnitude. To assess whether the discrepancy was caused by a systematic error, repeatability was assessed by replicating the standard controls at least three times. The relative abundance of the subgenomic mRNA for $N$ was higher than that of $R d R p$ during virus replication, consistent with previous observations (Lu et al., 2014), indicating that sensitivity was higher for the $N$ assay than the $R d R p$ assay. Although higher virus concentrations were generally observed for the $N$ assay compared to the $R d R p$ assay, three samples contained less virus in the $N$ assay (with $C t$ values abnormally exceeding 36). These results indicate that the $N$ assay is more sensitive and the $R d R p$ assay is more stable and accurate. The combination of these two assays could greatly reduce the falsepositive rate in future surveillance studies of SL-CoVs.

Bats in this unique cave excreted $10^{5}-10^{11}$ copies $/ g$ SL-CoVs from 2011 to 2014. The $N$ and $R d R p$ assays revealed dynamic changes in SL-CoV concentrations in the longitudinal surveillance. Fecal pellets with high virus concentrations were typically collected in either September 2012 or July 2013. Additionally, the average virus concentrations in July, September, and October were over 5-fold higher than those observed in April and May. These results indicated that SL-CoV amplification was more efficient from the late summer to autumn. Similar qRT-PCR results for other bat viruses, such as alphacoronaviruses (Drexler et al., 2011), henipaviruses (Chua et al., 2002), and filoviruses (Pourrut et al., 2007), have been reported. Bat-borne RNA viruses appear to have increased amplification and transmission efficiencies from the late summer to autumn, unlike DNA viruses (Drexler et al., 2011). Hypothetically, the higher concentrations of RNA viruses in bats at specific times may be related to the life habit of bats. Virus amplification after July may be associated with the establishment of a susceptible subpopulation of newborn bats who had not yet mounted their own adaptive immunity during the parturition period (Drexler et al., 2011). Our longitudinal survey of SL-CoVs in one bat population over 4 years provides valuable data for surveillance efforts to monitor the potential transmission of these viruses to humans.

\section{FOOTNOOTES}

This work was jointly funded by the National Natural Science Foundation of China (81290341), China Mega-Project for Infec- tious Disease (2014ZX10004001-003) from the Minister of Science and Technology of the People's Republic of China and USNIAID (R01AI110964). The authors declare that they have no conflict of interest. This article does not contain any studies with human or animal subjects performed by any of the authors.

Supplementary figures/tables are available on the website of Virologica Sinica: www.virosin.org; link.springer.com/journal/ 12250.

Mei-Niang Wang ${ }^{1}$, Wei Zhang ${ }^{1}$, Yu-Tao Gao ${ }^{1}$, Ben $\mathrm{Hu}^{1}$, Xing-Yi Ge ${ }^{1}$, Xing-Lou Yang ${ }^{1}$, Yun-Zhi Zhang ${ }^{2}$, Zheng-Li Shi ${ }^{1 \bowtie}$

1. Key Laboratory of Special Pathogens and Biosafety, Wuhan Institute of Virology, Chinese Academy of Sciences, Wuhan 430071, China

2. Yunnan Provincial Key Laboratory for Zoonosis Control and Prevention, Yunnan Institute of Endemic Diseases Control and Prevention, Dali 671000, China

$\triangle$ Correspondence:

Phone: +86-27-87197240, Email: zlshi@wh.iov.cn

ORCID: 0000-0001-8089-163X

Published online: 18 February 2016

\section{REFERENCES}

Chua KB, Koh CL, Hooi PS, et al. 2002. Microbes Infect, 4: 145-151.

Drexler JF, Corman VM, Wegner T, et al. 2011. Emerg Infect Dis, 17: 449-456.

Drexler JF, Gloza-Rausch F, Glende J, et al. 2010. J Virol, 84: 11336-11349.

Drosten C, Gunther S, Preiser W, et al. 2003. N Engl J Med, 348: 1967-1976.

Ge XY, Li JL, Yang XL, et al. 2013. Nature, 503: 535-538.

Li W, Shi Z, Yu M, et al. 2005. Science, 310: 676-679.

Lu XY, Whitaker B, Sakthivel SKK, et al. 2014. J Clin Microbiol, 52: $67-75$.

Menachery VD, Yount BL Jr., Debbink K, et al. 2015. Nat Med, 21:1508-1513.

Pourrut X, Delicat A, Rollin PE, et al. 2007. J Infect Dis, 196: S176-S183.

Tang XC, Zhang JX, Zhang SY, et al. 2006. J Virol, 80: 7481-7490.

Tong S, Conrardy C, Ruone S, et al. 2009. Emerg Infect Dis, 15: 482-485.

Vijaykrishna D, Smith GJD, Zhang JX, et al. 2007. J Virol, 81: 4012-4020.

Wang LF, Shi ZL, Zhang SY, et al. 2006. Emerg Infect Dis, 12: 1834-1840.

Yang XL, Tan B, Wang B, et al. 2015. J Gen Virol, doi: 10.1099/jgv. 0.000314 .

Yuan J, Hon CC, Li Y, et al. 2010. J Gen Virol, 91: 1058-1062. 